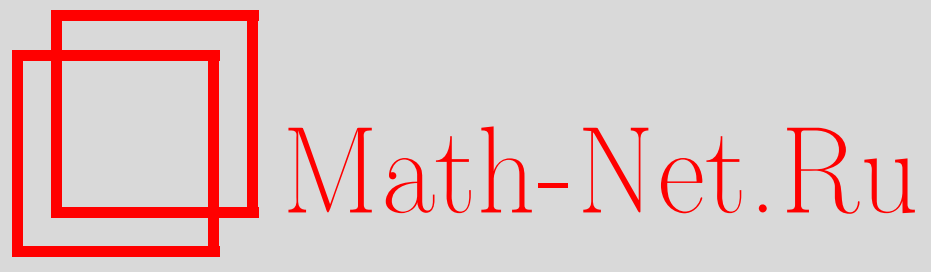

М. З. Гараев, В. С. Гарсиа, С. В. Конягин, Проблема Варинга с $\tau$-функцией Рамануджана, Изв. РАН. Сер. матем., 2008, том 72, выпуск 1, 39-50

DOI: https://doi.org/10.4213/im1139

Использование Общероссийского математического портала Math-Net.Ru подразумевает, что вы прочитали и согласны с пользовательским соглашением http://www . mathnet.ru/rus/agreement

Параметры загрузки:

IP : 54.84 .234 .179

26 апреля 2023 г., 09:16:06

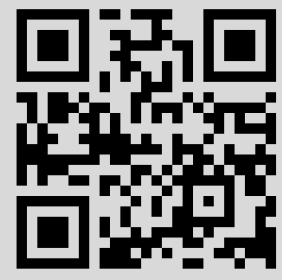


УДК 511.34

\author{
М. З. Гараев, В. К. Гарсия, С. В. Конягин
}

\title{
Проблема Варинга с $\tau$-функцией Рамануджана
}

Доказано, что диофантово уравнение $\sum_{i=1}^{74000} \tau\left(n_{i}\right)=N$, где $\tau(n)-$ $\tau$-функция Рамануджана, для любого целого $N$ имеет решение в натуральных числах $n_{1}, n_{2}, \ldots, n_{74000}$, удовлетворяющих условию $\max _{1 \leqslant i \leqslant 74000} n_{i} \ll$ $|N|^{2 / 11}+1$. Рассмотрены аналогичные вопросы в полях вычетов по модулю большого простого числа $p$.

Библиография: 13 наименований.

\section{§1. Введение}

Функция Рамануджана $\tau(n)$ определяется соотношением

$$
X \prod_{n=1}^{\infty}\left(1-X^{n}\right)^{24}=\sum_{n=1}^{\infty} \tau(n) X^{n}
$$

и обладает рядом замечательных свойств арифметической природы. Известно, что:

1) $\tau(n)$ - целочисленная мультипликативная функция, т. е. при $\operatorname{gcd}(n, m)=1$ имеем $\tau(n m)=\tau(n) \tau(m)$;

2) для любого целого числа $\alpha \geqslant 0$ и простого $q$ выполнено $\tau\left(q^{\alpha+2}\right)=$ $\tau\left(q^{\alpha+1}\right) \tau(q)-q^{11} \tau\left(q^{\alpha}\right)$, в частности $\tau\left(q^{2}\right)=\tau^{2}(q)-q^{11}$;

3) $\tau(n) \equiv \sum_{d \mid n} d^{11}(\bmod 691)$;

4) $\tau(n) \equiv \sum_{d \mid n} d^{11}\left(\bmod 2^{8}\right)$ при нечетном $n$;

5) $|\tau(q)| \leqslant 2 q^{11 / 2}$ для любого простого $q$ и $|\tau(n)| \ll n^{11 / 2+\varepsilon}$ для любого положительного целого $n$ и любого $\varepsilon>0$; это неравенство вывел П. Делинь [1].

Здесь и далее константы в символах Виноградова $\ll$ и 》 зависят только от $\varepsilon$.

Доказательства этих и других свойств функции $\tau(n)$ можно найти в работах [1]-[4]. В частности, из результатов, полученных в работе [4], можно вывести, что любой класс вычетов по модулю простого числа $p$ может быть записан как $\tau(n)(\bmod p)$ для некоторого положительного $n$. Используя оценки суммпроизведений, полученные Дж. Бургейном, Н. Кацем и Т. Тао [5], и оценки двойных тригонометрических сумм Виноградова, И. Е. Шпарлинский [6] установил, что значения $\tau(n), n \leqslant p^{4}$, образуют конечный аддитивный базис по модулю $p$, т. е. существует целая абсолютная константа $s \geqslant 1$ такая, что любой класс вычетов по модулю $p$ представи́м в виде

$$
\tau\left(n_{1}\right)+\cdots+\tau\left(n_{s}\right)(\bmod p),
$$

Работа третьего автора выполнена при поддержке ИНТАС (грант № 03-51-2070), РФФИ (грант № 03-51-5070) и Программы Президента РФ государственной поддержки ведущих научных школ (грант № НШ-5813.2006.1).

(C) М. З. ГАРАЕв, В.К. ГАРСИЯ, С. В. КоНЯГИН, 2008 
где $n_{1}, \ldots, n_{s} \leqslant p^{4}$ - некоторые целые числа. В настоящей работе, применяя новый подход, мы уменьшаем показатель степени 4 числа $p$ до наилучшего возможного значения, равного $2 / 11$.

Tеорема 1. Множество значений функции $\tau(n)$ образует конечный аддитивный базис множества цельх чисел. Более того, для любого целого числа $N$ диобантово уравнение

$$
\sum_{i=1}^{74000} \tau\left(n_{i}\right)=N
$$

имеет решение в натуральных числах $n_{1}, n_{2}, \ldots, n_{74000}$, удовлетворяющих условию

$$
\max _{1 \leqslant i \leqslant 74000} n_{i} \ll|N|^{2 / 11}+1 .
$$

Следует отметить, что число 74000 было получено в результате вычислений: $36 \times 2050+200$. Здесь число 2050 (так же, как число 200) может быть уменьшено, если использовать недавно полученные в работах [7], [8] улучшения в задаче Варинга-Гольдбаха. Однако количество слагаемых, тем не менее, останется большим. В связи с этим мы докажем следующий результат, в котором предполагается, что $p$ - большое простое число.

Теорема 2. Для любого иелого числа $\lambda$ сравнение

$$
\sum_{i=1}^{16} \tau\left(n_{i}\right)-\sum_{i=1}^{16} \tau\left(m_{i}\right) \equiv \lambda(\bmod p)
$$

имеет место для некоторых натуралъных чисел $n_{1}, m_{1}, \ldots, n_{16}, m_{16}$, удовлетворяющих условиям

$$
\max _{1 \leqslant i \leqslant 16}\left\{n_{i}, m_{i}\right\} \ll p^{2} \log ^{4} p, \quad \operatorname{gcd}\left(n_{i} m_{i}, 23 !\right)=1 .
$$

Используя теорему 2, мы показываем, что любой класс вычетов по модулю $p$ представим в виде

$$
\sum_{i=1}^{96} \tau\left(n_{i}\right)(\bmod p)
$$

при условии $\max _{1 \leqslant i \leqslant 96} n_{i} \ll p^{2} \log ^{4} p$. В частности, для некоторой положительной константы $C$ множество

$$
\left\{\tau(n)(\bmod p): n \leqslant C p^{2} \log ^{4} p\right\}
$$

образует конечный аддитивный базис в кольце вычетов $\mathbb{Z}_{p}$ порядка не более 96 (см. определение в [9]).

ТЕОрема 3. Для любого целого числа $\lambda$ и любого $\varepsilon>0$ сравнение

$$
\sum_{i=1}^{16} \tau\left(n_{i}\right) \equiv \lambda(\bmod p)
$$

разрешимо в натуральных числах $n_{1}, \ldots, n_{16}$, удовлетворяющих условию

$$
\max _{1 \leqslant i \leqslant 16} n_{i} \ll p^{3+\varepsilon} \text {. }
$$


В частности, множество $\left\{\tau(n)(\bmod p): n \leqslant p^{3+\varepsilon}\right\}$ является базисом в $\mathbb{Z}_{p}$ порядка не более 16 для всех достаточно больших простых $p$.

Далее через $q, q_{1}, q_{2}, \ldots$ обозначены простые чисела, через $|\mathscr{A}|$ - мощность множества $\mathscr{A}$.

\section{§ 2. Предварительные утверждения}

Сначала приведем следствие классического результата Хуа Ло-Кена [10].

Лемма 4. Пусть $s_{0}$ - биксированное целое число, не менъшее 2049, и Jчисло решений уравнения Варинга-Голъдбаха

$$
\sum_{i=1}^{s_{0}} q_{i}^{11}=N
$$

в простых числах $q_{1}, \ldots, q_{s_{0}}$ таких, что $q_{i}>23$ для всех $1 \leqslant i \leqslant s_{0}$. Тогда существуют положительные постоянные $c_{1}=c_{1}\left(s_{0}\right)$ и $c_{2}=c_{2}\left(s_{0}\right)$ такие, что для всех достаточно больших целых чисел $N, N \equiv s_{0}(\bmod 2)$, выполнены следующие ощенки:

$$
c_{1} \frac{N^{s_{0} / 11-1}}{(\log N)^{s_{0}}} \leqslant J \leqslant c_{2} \frac{N^{s_{0} / 11-1}}{(\log N)^{s_{0}}} .
$$

Далее нам понадобится следующий результат А. А. Глибичука [11].

Лемма 5. Если $\mathscr{X}, \mathscr{Y} \subset \mathbb{Z}_{p}-$ подмножества такие, что $|\mathscr{X}||\mathscr{Y}|>2 p$, mо

$$
\left\{\sum_{i=1}^{8} x_{i} y_{i}: x_{i} \in \mathscr{X}, y_{i} \in \mathscr{Y}\right\}=\mathbb{Z}_{p} .
$$

Понадобятся также некоторые результаты, основанные на значениях функции $\tau(n)$. По уже упомянутому результату Делиня имеем

$$
|\tau(n)| \ll n^{11 / 2+\varepsilon}
$$

и следующие $N^{6}$ чисел

$$
\sum_{i=1}^{6} \tau\left(a_{i}\right), \quad 1 \leqslant a_{1}, \ldots, a_{6} \leqslant N
$$

являются целыми числами порядка $O\left(N^{11 / 2+\varepsilon}\right)$. Таким образом, в среднем каждое число представимо в виде суммы шести значений функции $\tau(n)$ многими способами. Можно ожидать, что нуль тоже можно представить в виде суммы шести значений $\tau(n)$. Имея в виду это наблюдение, будем искать шесть положительных целых чисел $a_{1}, \ldots, a_{6}$, удовлетворяющих условию

$$
\sum_{i=1}^{6} \tau\left(a_{i}\right)=0
$$

Существует много формул, связывающих функцию $\tau(n)$ с функцией

$$
\sigma_{s}(n)=\sum_{d \mid n} d^{s} .
$$


Известно, например, что

$$
\tau(n)=\frac{65}{756} \sigma_{11}(n)+\frac{691}{756} \sigma_{5}(n)-\frac{691}{3} \sum_{k=1}^{n-1} \sigma_{5}(k) \sigma_{5}(n-k) .
$$

Другая формула имеет вид

$$
\tau(n)=n^{4} \sigma_{0}(n)-24 \sum_{k=1}^{n-1}\left(35 k^{4}-52 k^{3} n+18 k^{2} n^{2}\right) \sigma_{0}(k) \sigma_{0}(n-k)
$$

(см. [12]). Такого рода формулы полезны для численных расчетов значений функции $\tau(n)$. В частности, можно вывести, что

$$
\begin{aligned}
& \tau(12)=-370944, \quad \tau(27)=-73279080, \quad \tau(55)=2582175960, \\
& \tau(69)=4698104544, \quad \tau(90)=13173496560, \quad \tau(105)=-20380127040 \text {. }
\end{aligned}
$$

Таким образом, мы имеем

$$
\tau(12)+\tau(27)+\tau(55)+\tau(69)+\tau(90)+\tau(105)=0 .
$$

Далее, считая теорему 2 доказанной, умножим равенство множеств

$$
\left\{\sum_{i=1}^{16} \tau\left(n_{i}\right)-\sum_{i=1}^{16} \tau\left(m_{i}\right)(\bmod p): m_{i}, n_{i} \leqslant C p^{2} \log ^{4} p, \operatorname{gcd}\left(m_{i} n_{i}, 23 !\right)=1\right\}=\mathbb{Z}_{p}
$$

на $\tau(12)$. Поскольку

$$
\begin{aligned}
& \tau(12)\left(\sum_{i=1}^{16} \tau\left(n_{i}\right)-\sum_{i=1}^{16} \tau\left(m_{i}\right)\right)=\sum_{i=1}^{16} \tau(12) \tau\left(n_{i}\right) \\
& +(\tau(27)+\tau(55)+\tau(69)+\tau(90)+\tau(105)) \sum_{i=1}^{16} \tau\left(m_{i}\right)
\end{aligned}
$$

то, используя мультипликативность функции $\tau(n)$, мы выводим, что множество

$$
\left\{\tau(n)(\bmod p): n \leqslant C p^{2} \log ^{4} p\right\}
$$

является аддитивным базисом в $\mathbb{Z}_{p}$ порядка не более 96.

Полезно также отметить, что

$$
\begin{gathered}
\tau(6)=-6048, \quad \tau(14)=401856, \quad \tau(29)=128406630, \quad \tau(41)=308120442, \\
\tau(42)=101267712, \quad \tau(44)=-786948864, \quad \tau(48)=248758272 .
\end{gathered}
$$

Таким образом, получим представление нуля в виде суммы семи значений функции $\tau(n)$ :

$$
\tau(6)+\tau(14)+\tau(29)+\tau(41)+\tau(42)+\tau(44)+\tau(48)=0 .
$$




\section{§ 3. Доказательство теоремы 1}

Пусть $M-$ большой четный параметр. Определим следующее множество:

$$
\mathscr{Q}=\left\{q: 23<q \leqslant M^{1 / 11}\right\} .
$$

Подмножество $\mathscr{Q}^{\prime} \subset \mathscr{Q}$ назовем допустимым, если уравнение

$$
\sum_{i=1}^{6} \tau\left(q_{i}^{\prime}\right)=\sum_{i=7}^{12} \tau\left(q_{i}^{\prime}\right)
$$

не имеет решенеий $q_{1}^{\prime}, \ldots, q_{12}^{\prime} \in \mathscr{Q}^{\prime}$, удовлетворяющих условиям

$$
q_{1}^{\prime}<\cdots<q_{6}^{\prime}, \quad q_{7}^{\prime}<\cdots<q_{12}^{\prime}, \quad\left(q_{1}^{\prime}, \ldots, q_{6}^{\prime}\right) \neq\left(q_{7}^{\prime}, \ldots, q_{12}^{\prime}\right) .
$$

Используя различные свойства функции $\tau(n)$, легко проверить, что существуют допустимые подмножества из 12 элементов. Это можно получить, используя упомянутые выше сравнения

$$
\tau(q) \equiv 1+q^{11}(\bmod 691), \quad \tau(q) \equiv 1+q^{11}\left(\bmod 2^{8}\right),
$$

где $q$ - нечетое простое число, китайскую теорему об остатках и теорему о простых числах в арифметической прогрессии для доказательства того, что для любого $j, 1 \leqslant j \leqslant 12$, можно найти достаточно большое простое число $\ell_{j}$ такое, что

$$
\tau\left(\ell_{j}\right) \equiv 2^{j}(\bmod 8 \times 691) .
$$

Далее, рассмотрим допустимое подмножество $\mathscr{Q}^{\prime}$ наибольшей мощности. Если их несколько, то в качестве $\mathscr{Q}^{\prime}$ выберем любое из таких подмножеств. В частности, $\left|\mathscr{Q}^{\prime}\right| \geqslant 12$, и все суммы вида

$$
\sum_{i=1}^{6} \tau\left(q_{i}^{\prime}\right), \quad q_{1}^{\prime}<\cdots<q_{6}^{\prime}, \quad q_{1}^{\prime}, \ldots, q_{6}^{\prime} \in \mathscr{Q}^{\prime}
$$

различны. Из оценки Делиня функции $\tau(q)$ и принципа Дирихле выводим:

$$
\left|\mathscr{Q}^{\prime}\right|^{6} \ll\left(M^{1 / 11}\right)^{11 / 2},
$$

откуда получаем

$$
\left|\mathscr{Q}^{\prime}\right| \ll M^{1 / 11-1 / 132} .
$$

Для данного $q \in \mathscr{Q} \backslash \mathscr{Q}^{\prime}$ рассмотрим множество $\mathscr{Q}^{\prime} \cup\{q\}$. Из максимальности $\left|\mathscr{Q}^{\prime}\right|$ следует существование $q_{1}^{\prime}, \ldots, q_{12}^{\prime} \in \mathscr{Q}^{\prime} \cup\{q\}$ таких, что

$$
\begin{gathered}
\sum_{i=1}^{6} \tau\left(q_{i}^{\prime}\right)=\sum_{i=7}^{12} \tau\left(q_{i}^{\prime}\right), \\
q_{1}^{\prime}<\cdots<q_{6}^{\prime}, \quad q_{7}^{\prime}<\cdots<q_{12}^{\prime}, \quad\left(q_{1}^{\prime}, \ldots, q_{6}^{\prime}\right) \neq\left(q_{7}^{\prime}, \ldots, q_{12}^{\prime}\right) .
\end{gathered}
$$

Из определения множества $\mathscr{Q}^{\prime}$ получаем, что

$$
q \in\left\{q_{1}^{\prime}, \ldots, q_{12}^{\prime}\right\}
$$


Кроме того, согласно (6) число $q$ появляется в последовательности $q_{1}^{\prime}, \ldots, q_{12}^{\prime}$ не более чем в двух местах. Если элемент $q$ появляется там дважды, то он появляется ровно один раз в последовательности $q_{1}^{\prime}, \ldots, q_{6}^{\prime}$ и ровно один раз в последовательности $q_{7}^{\prime}, \ldots, q_{12}^{\prime}$. Следовательно, можно вычесть из обеих частей $(5)$ число $\tau(q)$ и перенумеровать оставшиеся числа $q_{i}^{\prime}$. Мы получим существование элементов

$$
q_{1}^{\prime}, \ldots, q_{5}^{\prime} \in \mathscr{Q}^{\prime}, \quad q_{7}^{\prime}, \ldots, q_{11}^{\prime} \in \mathscr{Q}^{\prime}
$$

таких, что

$$
\begin{gathered}
\sum_{i=1}^{5} \tau\left(q_{i}^{\prime}\right)=\sum_{i=7}^{11} \tau\left(q_{i}^{\prime}\right), \\
q_{1}^{\prime}<\cdots<q_{5}^{\prime}, \quad q_{7}^{\prime}<\cdots<q_{11}^{\prime}, \quad\left(q_{1}^{\prime}, \ldots, q_{5}^{\prime}\right) \neq\left(q_{7}^{\prime}, \ldots, q_{11}^{\prime}\right) .
\end{gathered}
$$

Поскольку $\left|\mathscr{Q}^{\prime}\right|>10$, то найдется число

$$
q^{\prime} \in \mathscr{Q}^{\prime} \backslash\left\{q_{1}^{\prime}, \ldots, q_{5}^{\prime}, q_{7}^{\prime}, \ldots, q_{11}^{\prime}\right\}
$$

Тогда

$$
\sum_{i=1}^{5} \tau\left(q_{i}^{\prime}\right)+\tau\left(q^{\prime}\right)=\sum_{i=7}^{11} \tau\left(q_{i}^{\prime}\right)+\tau\left(q^{\prime}\right) .
$$

Это ввиду соотношений (7)-(9) противоречит определению $\mathscr{Q}^{\prime}$.

Таким образом, единственный возможный случай - это когда $q$ появляется в последовательности $q_{1}^{\prime}, \ldots, q_{12}^{\prime}$ ровно один раз. Значит, в силу (5) существуют $\tilde{q}_{1}, \ldots, \tilde{q}_{11} \in \mathscr{Q}^{\prime}$ такие, что

$$
\sum_{i=1}^{6} \tau\left(\tilde{q}_{i}\right)=\sum_{i=7}^{11} \tau\left(\tilde{q}_{i}\right)+\tau(q) .
$$

Следовательно, для всех $q \in \mathscr{Q} \backslash \mathscr{Q}^{\prime}$ мы получаем

$$
\begin{aligned}
\sum_{i=1}^{6} \tau\left(\tilde{q}_{i} q\right)-\sum_{i=7}^{11} \tau\left(\tilde{q}_{i} q\right)-\tau\left(q^{2}\right) & =\tau(q) \sum_{i=1}^{6} \tau\left(\tilde{q}_{i}\right)-\tau(q) \sum_{i=7}^{11} \tau\left(\tilde{q}_{i}\right)-\tau\left(q^{2}\right) \\
& =\tau^{2}(q)-\tau\left(q^{2}\right)=q^{11}
\end{aligned}
$$

Таким образом, мы доказали, что для любого элемента $q \in \mathscr{Q} \backslash \mathscr{Q}^{\prime}$ существуют элементы $\tilde{q}_{1}, \ldots, \tilde{q}_{11} \in \mathscr{Q}^{\prime}$ такие, что

$$
q^{11}=\sum_{i=1}^{6} \tau\left(\tilde{q}_{i} q\right)-\sum_{i=7}^{11} \tau\left(\tilde{q}_{i} q\right)-\tau\left(q^{2}\right) .
$$

Наша следующая цель - доказать разрешимость уравнения Варинга-Гольдбаха

$$
\sum_{j=1}^{2050} q_{j}^{11}=M
$$

в простых числах $q_{1}, \ldots, q_{2050} \in \mathscr{Q} \backslash \mathscr{Q}^{\prime}$. Сначала заметим, что из соотношения (11) вытекает неравенство $q_{j} \leqslant M^{1 / 11}$. Далее, для числа решений $I$ 
уравнения (11) с условием $q_{j} \in \mathscr{Q}$ из леммы 4 при $s_{0}=2050$ мы получаем следующую оценку снизу:

$$
I \geqslant c_{1} \frac{M^{2050 / 11-1}}{(\log M)^{2050}} .
$$

Для числа решений $J$ уравнения (11) таких, что по крайней мере одно $q_{j_{0}} \in \mathscr{Q}^{\prime}$, из леммы 4 при $s_{0}=2049$ и (4) получаем оценку сверху:

$$
J \leqslant 2050 c_{2}\left|\mathscr{Q}^{\prime}\right| \frac{M^{2049 / 11-1}}{(\log M)^{2049}} \ll \frac{M^{2050 / 11-1-1 / 132}}{(\log M)^{2049}} .
$$

Таким образом, $I>J$. Следовательно, уравнение (11) разрешимо в числах $q_{j} \in$ $\mathscr{Q} \backslash \mathscr{Q}^{\prime}$. Зафиксируем одно из таких решений $q_{1}, \ldots, q_{2050} . \mathrm{K}$ каждому $q_{j}, 1 \leqslant$ $j \leqslant 2050$, применим (10) при $q=q_{j}$ и просуммируем полученные соотношения по $1 \leqslant j \leqslant 2050$. Так как $\tilde{q}_{i} q \leqslant M^{2 / 11}$, то мы получаем

$$
M=\sum_{i=1}^{6 \times 2050} \tau\left(n_{i}\right)-\sum_{i=1}^{6 \times 2050} \tau\left(m_{i}\right)
$$

где

$$
\max _{1 \leqslant i \leqslant 6 \times 2050}\left\{n_{i}, m_{i}\right\} \leqslant M^{2 / 11}, \quad \operatorname{gcd}\left(n_{i} m_{i}, 23 !\right)=1 .
$$

Мы предположили, что $M$ - большое четное число. Ясно, что можно умножить обе части равенства (14) на -1 и получить представление такого же вида и для $-M$. Более того, мы можем рассматривать $M$ любой четности, для этого следует заменить число $M$ на $M-\tau(1)$ или $M-\tau(29)$. Таким образом, любое целое число $M$ с достаточно большим $|M|$ может быть представлено в виде

$$
M=\sum_{i=1}^{6 \times 2050} \tau\left(n_{i}\right)-\sum_{i=1}^{6 \times 2050+1} \tau\left(m_{i}\right)
$$

где

$$
\max _{1 \leqslant i \leqslant 6 \times 2050+1}\left\{n_{i}, m_{i}\right\} \leqslant|M|^{2 / 11}+1, \quad \operatorname{gcd}\left(n_{i} m_{i}, 23 !\right)=1 .
$$

Напомним, что (см. (1))

$$
-\tau(12)=370944=\tau(27)+\tau(55)+\tau(69)+\tau(90)+\tau(105) .
$$

Следовательно, умножая (15) на $-\tau(12)$ и используя мультипликативность функции $\tau(n)$, получаем

$$
370944 M=\sum_{i=1}^{6 \times 6 \times 2050+1} \tau\left(n_{i}\right)
$$

при условии $\max _{1 \leqslant i \leqslant 6 \times 6 \times 2050+1} n_{i} \leqslant 106|M|^{2 / 11}$.

Докажем, что любое целое число $r, 0 \leqslant r<370944$, может быть представлено в виде суммы, скажем, ровно 198 чисел вида $\tau(n), n \leqslant 105$ (дополнительные вычисления могут уменьшить 198 до значительно меньшей константы, но это существенно не повлияет на наш результат). Для этого напомним, что

$$
\tau(1)=1, \quad \tau(2)=-24, \quad \tau(3)=252, \quad \tau(5)=4830, \quad \tau(8)=84480 .
$$


Если $0 \leqslant r<370944$, то

$$
r=84480 r_{5}+r_{4}^{\prime}=\tau(8) r_{5}+r_{4}^{\prime}
$$

для некоторых целых чисел $0 \leqslant r_{5} \leqslant 4$ и $0 \leqslant r_{4}^{\prime}<84480$. Далее, любое такое число $r_{4}^{\prime}$ может быть записано в виде

$$
r_{4}^{\prime}=4830 r_{4}+r_{3}^{\prime}=\tau(5) r_{4}+r_{3}^{\prime},
$$

где $0 \leqslant r_{4} \leqslant 17$ и $0 \leqslant r_{3}^{\prime}<4830$. Любое такое число $r_{3}^{\prime}$ можно представить в виде

$$
r_{3}^{\prime}=252 r_{3}-r_{2}^{\prime}=\tau(3) r_{3}-r_{2}^{\prime},
$$

где $0 \leqslant r_{3} \leqslant 20$ и $0 \leqslant r_{2}^{\prime}<252$. Любое такое число $r_{2}^{\prime}$ записывается в виде

$$
r_{2}^{\prime}=24 r_{2}-r_{1}=-\tau(2) r_{2}-r_{1},
$$

где $0 \leqslant r_{2} \leqslant 11$ и $0 \leqslant r_{1}<24$. Таким образом, мы получаем

$$
r=\tau(8) r_{5}+\tau(5) r_{4}+\tau(3) r_{3}+\tau(2) r_{2}+r_{1} .
$$

Следовательно, чтобы представить данное $r$, достаточно использовать

$$
r_{5}+\cdots+r_{1} \leqslant 75
$$

слагаемых вида $\tau(n), n \leqslant 10$. С другой стороны, любое целое число, большее 29 , может быть записано в форме $6 x+7 y$ с неотрицательными целыми $x, y$. Таким образом, чтобы получить фиксированное количество слагаемых для всех $r$, можно использовать конструкции (1) и (2). В частности, любое целое $r, 0 \leqslant$ $r<370944$, можно представить в виде

$$
\sum_{i=1}^{198} \tau\left(a_{i}\right)
$$

с натуральными числами $a_{1}, \ldots, a_{198} \leqslant 105$.

Пусть теперь $N$ будет произвольным целым числом с достаточно большим модулем $|N|$. Последнее рассуждение показывает, что для некоторых натуральных чисел $a_{1}, \ldots, a_{199} \leqslant 105$ мы получаем

$$
N \equiv \sum_{i=1}^{198} \tau\left(a_{i}\right)(\bmod 370944)
$$

Следовательно, используя (16), выводим:

$$
N=\sum_{i=1}^{198} \tau\left(a_{i}\right)+370944 M=\sum_{i=1}^{6 \times 6 \times 2050+199} \tau\left(n_{i}\right)=\sum_{i=1}^{73999} \tau\left(n_{i}\right),
$$

где

$$
\max _{1 \leqslant i \leqslant 73999} n_{i} \leqslant 106|M|^{2 / 11} \leqslant 15 N^{2 / 11}
$$

Таким образом, мы доказали, что существует абсолютная положительная целая константа $N_{0}$ такая, что для любого целого числа $N,|N| \geqslant N_{0}$, уравнение

$$
\sum_{i=1}^{73999} \tau\left(n_{i}\right)=N
$$


имеет решение в натуральных числах $n_{1}, \ldots, n_{73999} \ll|N|^{2 / 11}$.

Пусть теперь $N-$ произвольное целое число. Если $|N|>N_{0}$, то $|N-\tau(1)| \geqslant$ $N_{0}$, и поэтому мы можем выразить число $N-\tau(1)$ в виде суммы 73999 значений $\tau(n)$ при $n \ll|N|^{2 / 11}$. В этом случае теорема 1 доказана.

Если $|N| \leqslant N_{0}$, то возьмем постоянное целое число $n_{0}$ такое, что $\left|\tau\left(n_{0}\right)\right|>$ $2 N_{0}$. Тогда

$$
\left|N-\tau\left(n_{0}\right)\right|>N_{0} .
$$

Таким образом, $N-\tau\left(n_{0}\right)$ может быть представлено в виде суммы 73999 значений $\tau(n), n \ll 1$. Теорема 1 доказана.

ЗАмечАниЕ 1. Легко видеть, что построенные в доказательстве теоремы 1 числа $n_{i}$ удовлетворяют условию

$$
\tau\left(n_{i}\right) \ll|N|
$$

ЗАмЕчАНИЕ 2. В нашей последующей статье мы докажем, что для любого целого числа $N,|N| \geqslant 2$, диофантово уравнение

$$
\sum_{i=1}^{148000} \tau\left(n_{i}\right)=N
$$

имеет решение в натуральных числах $n_{1}, n_{2}, \ldots, n_{148000}$, удовлетворяющих условию

$$
\max _{1 \leqslant i \leqslant 148000} n_{i} \ll|N|^{2 / 11} e^{-c \log |N| / \log \log |N|}
$$

для некоторой абсолютной константы $c>0$. Вследствие оценки Делиня имеем $\tau(n) \leqslant n^{11 / 2} d(n)$, где $d(n)$ - число делителей $n$, это условие соответствует наилучшей возможной границе для размера переменных $n_{i}$ с точностью до значения константы $c$.

\section{§4. Доказательство теоремы 2}

Пусть $C$ - большая положительная константа. Определим множества

$$
\begin{gathered}
\mathscr{Q}=\left\{q: 23<q \leqslant C p^{1 / 2} \log p\right\}, \\
\mathscr{I}=\{\tau(q)(\bmod p): q \in \mathscr{Q}\} .
\end{gathered}
$$

Если $|\mathscr{I}|>3 \sqrt{p}$, то можно разбить $\mathscr{I}$ на два подмножества $\mathscr{X}, \mathscr{Y}$ таких, что $|\mathscr{X}||\mathscr{Y}|>2 p$. Доказываемый результат в этом случае следует из леммы 5.

Теперь рассмотрим случай $|\mathscr{I}|<3 \sqrt{p}$. Тогда

$$
\mathscr{Q}=\bigcup_{i=1}^{|\mathscr{I}|} \mathscr{A}_{i},
$$

где множества $\mathscr{A}_{i}$ определяются так, что условие $q^{\prime}, q^{\prime \prime} \in \mathscr{A}_{i}$ влечет сравнение $\tau\left(q^{\prime}\right) \equiv \tau\left(q^{\prime \prime}\right)(\bmod p)$.

Ясно, что существует подмножество $\mathscr{A}_{i}^{\prime} \subset \mathscr{A}_{i}$ такое, что

$$
0 \leqslant\left|\mathscr{A}_{i}\right|-\left|\mathscr{A}_{i}^{\prime}\right| \leqslant 3, \quad\left|\mathscr{A}_{i}^{\prime}\right| \equiv 0(\bmod 4) .
$$


Обозначим

$$
\mathscr{Q}^{\prime}=\bigcup_{i=1}^{|\mathscr{I}|} \mathscr{A}_{i}^{\prime}
$$

По теореме о распределении простых чисел мы имеем $|\mathscr{Q}| \geqslant C p^{1 / 2}$, если $p$ достаточно велико. Тогда

$$
\left|\mathscr{Q}^{\prime}\right| \geqslant|\mathscr{Q}|-3|\mathscr{I}| \geqslant|\mathscr{Q}|-9 p^{1 / 2} \geqslant(C-9) p^{1 / 2}
$$

Поскольку мощность каждого множества $\mathscr{A}_{i}^{\prime}$ четна, можно составить $\left|\mathscr{A}_{i}^{\prime}\right| / 2$ пар различных простых из $\mathscr{A}_{i}^{\prime}$. Тогда всего будет

$$
\sum_{i=1}^{|\mathscr{I}|}\left|\mathscr{A}_{i}^{\prime}\right| / 2=\left|\mathscr{Q}^{\prime}\right| / 2
$$

пар $\left(q, q^{\prime}\right)$. Разделим это множество пар на два непересекающихся подмножества $J_{1}$ и $J_{2}$ так, чтобы $\left|J_{1}\right|=\left|J_{2}\right|=\left|\mathscr{Q}^{\prime}\right| / 4$. Далее рассмотрим следующие два множества:

$$
\begin{aligned}
\mathscr{X} & =\left\{\tau\left(q q^{\prime}\right)-\tau\left(q^{2}\right)(\bmod p):\left(q, q^{\prime}\right) \in J_{1}\right\}, \\
\mathscr{Y} & =\left\{\tau\left(q q^{\prime}\right)-\tau\left(q^{2}\right)(\bmod p):\left(q, q^{\prime}\right) \in J_{2}\right\}
\end{aligned}
$$

Поскольку

$$
\tau\left(q q^{\prime}\right)-\tau\left(q^{2}\right)=\tau(q) \tau\left(q^{\prime}\right)-\tau\left(q^{2}\right) \equiv \tau^{2}(q)-\tau\left(q^{2}\right) \equiv q^{11}(\bmod p)
$$

и $q^{11}(\bmod p)$ может принимать любое значение не более 11 раз, то получаем

$$
|\mathscr{X}| \geqslant\left|J_{1}\right| / 11=\left|\mathscr{Q}^{\prime}\right| / 44, \quad|\mathscr{Y}| \geqslant\left|J_{2}\right| / 11 \geqslant\left|\mathscr{Q}^{\prime}\right| / 44
$$

Следовательно, если выбрать, например, $C=100$, то из (17) получим, что $|\mathscr{X}||\mathscr{Y}|>2 p$. Применяя лемму 5 , мы завершим доказательство теоремы 2.

\section{§5. Доказательство теоремы 3}

Рассмотрим множество классов вычетов

$$
\mathscr{A}^{\prime}=\{\tau(q)(\bmod p): p / 2<q \leqslant p\} .
$$

Для заданного $a^{\prime} \in \mathscr{A}^{\prime}$ пусть $I\left(a^{\prime}\right)$ - число решений сравнения

$$
\tau(q) \equiv a^{\prime}(\bmod p), \quad p / 2<q \leqslant p .
$$

Из асимптотического закона распределения простых чисел имеем

$$
\sum_{a^{\prime} \in \mathscr{A}^{\prime}} I\left(a^{\prime}\right)=\sum_{p / 2<q \leqslant p} 1 \gg p \log ^{-1} p .
$$

Следовательно, существует элемент $a_{0}^{\prime} \in \mathscr{A}^{\prime}$ такой, что

$$
I\left(a_{0}^{\prime}\right) \gg p\left|\mathscr{A}^{\prime}\right|^{-1} \log ^{-1} p .
$$


Далее, обозначим $\mathscr{A}=\mathscr{A}^{\prime} \backslash\left\{a_{0}^{\prime}\right\}$, если $\left|\mathscr{A}^{\prime}\right| \geqslant 2$, и $\mathscr{A}=\{\tau(1)\}=\{1\}$, если $\left|\mathscr{A}^{\prime}\right|=1$. Тогда

$$
\left|\mathscr{A}^{\prime}\right| / 2 \leqslant|\mathscr{A}| \leqslant\left|\mathscr{A}^{\prime}\right| \text {. }
$$

Теперь определим множество

$$
\mathscr{B}=\left\{\tau\left(q^{2}\right)(\bmod p): p / 2<q \leqslant p, \tau(q) \equiv a_{0}^{\prime}(\bmod p)\right\} .
$$

Элементы множества $\mathscr{B}$ имеют вид

$$
\tau\left(q^{2}\right)=\tau^{2}(q)-q^{11} \equiv\left(a_{0}^{\prime}\right)^{2}-q^{11}(\bmod p),
$$

где $p / 2<q \leqslant p$ и $\tau(q) \equiv a_{0}^{\prime}(\bmod p)$. Таким образом, в силу $(18)$ и $(19)$ число $q$ может принимать любое значение из множества, содержащего

$$
\gg p\left|\mathscr{A}^{\prime}\right|^{-1} \log ^{-1} p \gg p|\mathscr{A}|^{-1} \log ^{-1} p
$$

различных классов вычетов по модулю $p$. Так как $q^{11}(\bmod p)$ может принимать любое значение не более 11 раз, то

$$
|\mathscr{B}| \gg p|\mathscr{A}|^{-1} \log ^{-1} p .
$$

Возьмем произвольное $\varepsilon, 0<\varepsilon<0.1$. Пусть $\mathscr{C}$ - множество попарно различных элементов последовательности

$$
\tau(q)(\bmod p), \quad \tau\left(q^{2}\right)(\bmod p),
$$

где $q \leqslant p^{0.5 \varepsilon}$. Применяя приведенное выше рассуждение ко множествам

$$
\begin{gathered}
\left\{\tau(q)(\bmod p): q \leqslant p^{0.5 \varepsilon}\right\}, \\
\left\{\tau\left(q^{2}\right)(\bmod p): q \leqslant p^{0.5 \varepsilon}\right\},
\end{gathered}
$$

получаем, что $|\mathscr{C}| \gg p^{\varepsilon / 6}$. Заметим, что множества $\mathscr{A}, \mathscr{B}, \mathscr{C}$ составлены из элементов вида $\tau\left(n_{1}\right), \tau\left(n_{2}\right), \tau\left(n_{3}\right)$ таким образом, что числа $n_{1}, n_{2}, n_{3}$ попарно взаимно просты и $n_{1} \leqslant p, n_{2} \leqslant p^{2}, n_{3} \leqslant p^{\varepsilon}$.

Если $|\mathscr{A}|<p^{0.1 \varepsilon}$, то $|\mathscr{B}| \gg p^{1-\varepsilon / 9}$ в силу $(20)$, и поэтому $|\mathscr{B}||\mathscr{C}| \gg p^{1+0.01 \varepsilon}$. Следовательно, мы можем применить лемму 5 при $\mathscr{X}=\mathscr{B}$ и $\mathscr{Y}=\mathscr{C}$ и использовать мультипликативность функции $\tau(n)$. Элементы множества

$$
\mathscr{X} \mathscr{Y}=\{x y: x \in \mathscr{X}, y \in \mathscr{Y}\}
$$

в этом случае будут иметь вид $\tau(n)(\bmod p)$, где $n \leqslant p^{2+\varepsilon}$.

Если $|\mathscr{A}|>p^{2 / 3}$, то мы разобьем $\mathscr{A}$ на два непересекающихся подмножества $\mathscr{A}_{1}$ и $\mathscr{A}_{2}$, имеющих не меньше $p^{2 / 3} / 3$ элементов в каждом. Применим лемму 5 для $\mathscr{X}=\mathscr{A}_{1}$ и $\mathscr{Y}=\mathscr{A}_{2}$. Элементы множества $\mathscr{X} \mathscr{Y}$ в этом случае будут иметь вид $\tau(n)(\bmod p)$, где $n \leqslant p^{2}$.

Если $p^{\varepsilon / 10} \leqslant \mathscr{A} \leqslant p^{2 / 3}$, то через $\mathscr{T}$ обозначим то из двух множеств $\mathscr{A}+\mathscr{C}$ и $\mathscr{A} \mathscr{C}$, которое имеет наибольшую мощность. Так как $|\mathscr{C}| \gg p^{\varepsilon / 6}$, то по оценке Бургейна [13, теорема 1.1] существует положительная константа $\gamma=\gamma(\varepsilon)>0$ такая, что

$$
|\mathscr{T}| \gg p^{\gamma}|\mathscr{A}| \text {. }
$$

Тогда $|\mathscr{B}||\mathscr{T}| \gg p^{1+\gamma / 2}$. Поэтому мы можем применить лемму 5 для $\mathscr{X}=\mathscr{B}$ и $\mathscr{Y}=\mathscr{T}$. Элементы множества $\mathscr{X} \mathscr{Y}$ в этом случае будут иметь вид либо 
$\tau\left(n_{1}\right)+\tau\left(n_{2}\right)(\bmod p)$ при $n \leqslant p^{3}$, либо $\tau(n)(\bmod p)$ при $n \leqslant p^{3+\varepsilon}$. Отсюда вытекает теорема 3 .

\section{Список литературы}

1. П. Делинь, "Гипотеза Вейля. I", УМН, 30:5 (1975), 159-190; пер. с фр.: P. Deligne, "La conjecture de Weil. I", Inst. Hautes Études Sci. Publ. Math., 1974, № 43, 273-307.

2. H. Iwaniec, Topics in clasical automorphic forms, Grad. Stud. Math., Amer. Math. Soc., Providence, RI, 1997.

3. Н. Коблиц, Введение в эллиптические кривые и модулярные формы, ред. Ю.И. Манин, Мир, М., 1988; пер. с англ.: N. Koblitz, Introduction to elliptic curves and modular forms, Grad. Texts in Math., 97, Springer-Verlag, New York, 1984.

4. Ж.П. Серр, "Сравнения и модулярные формы”, УМН, 28:2 (1973), 183-196; пер. с фр.: J.-P. Serre, "Congruences et formes modulaires [d'après H.P.F. Swinnerton-Dyer]", Séminaire Bourbaki, 24e année (1971/1972), Exp. No. 416, Lecture Notes in Math., 317, Springer, Berlin, 1973, 319-338.

5. J. Bourgain, N. Katz, T. Tao, "A sum-product estimate in finite fileds and applications", Geom. Func. Anal., 14:1 (2004), 27-57.

6. I. E. Shparlinski, "On the value set of the Ramanujan function", Arch. Math., 85:6 (2005), 508-513.

7. Г. И. Архипов, В.Н. Чубариков, "О числе слагаемых в аддитивной проблеме Виноградова и ее обобщениях", IV Международная конферениия "Современные проблемы теории чисел и ее приложения": Актуальные проблемы, Ч. I (Тула, 10-15 сентября 2001), Мех.-мат. МГУ им. М. В. Ломоносова, М., 2002, 5-38.

8. A. V. Kumchev, D. I. Tolev, "An invitation to additive prime number theory", Serdica Math. J., 31:1-2 (2005), 1-74.

9. А. А. Карацуба, "Аддитивные сравнения", Изв. РАН. Сер. матем., 61:2 (1997), 81-94; англ. пер.: А. A. Karatsuba, "Additive congruences", Izv. Math., 61:2 (1997), $317-329$.

10. Хуа Ло-Кен, Аддитивная теория простых чисел, Тр. МИАН, 22, 1947.

11. А. А. Глибичук, "Комбинаторные свойства множеств вычетов по простому модулю и задача Эрдёша-Грэхэма", Матем. заметки, 79:3 (2006), 384-395; англ. пер.: A. A. Glibichuk, "Combinational properties of sets of residues modulo a prime and the Erdős-Graham problem", Math. Notes, 79:3-4 (2006), 356-365.

12. D. Niebur, "A formula for Ramanujan's $\tau$-function", Illinois J. Math., 19 (1975), 448-449.

13. J. Bourgain, "More on the sum-product phenomenon in prime fields and its applications", Int. J. Number Theory, 1:1 (2005), 1-32.

M. 3. Гараeb (M. Z. Garaev)

National Autonomous University of Mexico,

Поступило в редакцию

Institute of Mathematics

B. К. ГАРсия (V. C. Garcia)

National Autonomous University of Mexico,

Institute of Mathematics

C. В. Конягин (S. V. Konyagin)

Московский государственный университет

им. М. В. Ломоносова,

механико-математический факультет 